\title{
RIEMANN STEP FUNCTION APPROXIMATION OF BOCHNER INTEGRABLE FUNCTIONS ${ }^{1}$
}

\author{
M. A. FREEDMAN
}

\begin{abstract}
Let $L^{1}(0, T ; X)$ denote the space of all Bochner integrable functions $f$ which map the interval $[0, T]$ into the Banach space $X$. Then we show that $f$ is the uniform limit in the $L^{1}$-norm of its Riemann step function approximations along nearly every sequence of partitions of $[0, T]$ with mesh size approaching zero.
\end{abstract}

1. Convergence of the Bochner integral. As every student of calculus knows, given a function $f(\cdot)$ continuous on the interval $[0, T]$, the integral of $f$ exists and is given by the limit of Riemann sum approximations:

$$
\int_{0}^{T} f(t) d t=\lim _{n \rightarrow \infty} \sum_{k=1}^{n} f(k T / n) \frac{T}{n}
$$

In fact, let $\left\{P^{n}\right\}_{n=1}^{\infty}$ be any sequence of partitions of $[0, T]$. Say each $P^{n}$ is given by

with mesh size

$$
P^{n}: 0=t_{0}^{n}<t_{1}^{n}<\cdots<t_{N_{n}}^{n}=T,
$$

$$
\left\|P^{n}\right\|=\max _{1 \leq k \leq N_{n}}\left(t_{k}^{n}-t_{k-1}^{n}\right),
$$

and suppose that $\left\|P^{n}\right\| \rightarrow 0$ as $n \rightarrow \infty$. Then for any choice of intermediate points $\xi_{k}^{n} \in\left(t_{k-1}^{n}, t_{k}^{n}\right]$, the integral of $f$ satisfies

$$
\lim _{n \rightarrow \infty}\left|\int_{0}^{T} f(t) d t-\sum_{k=1}^{N_{n}} f\left(\xi_{k}^{n}\right)\left(t_{k}^{n}-t_{k-1}^{n}\right)\right| \leq \lim _{n \rightarrow \infty} \sum_{k=1}^{N_{n}} \int_{t_{k-1}^{n}}^{t_{k}^{n}}\left|f(t)-f\left(\xi_{k}^{n}\right)\right| d t=0 .
$$

Hence the Riemann step functions for $f$, given by

$$
w_{n}(t)=\sum_{k=1}^{N_{n}} f\left(\xi_{k}^{n}\right) I_{\left(t_{k-1}^{n}, t_{k}^{n}\right]}(t), \text { satisfy } \lim _{n \rightarrow \infty} \int_{0}^{T}\left|f(t)-w_{n}(t)\right| d t=0 .
$$

Here $I_{A}(t)$ denotes the indicator function of the set $A$. When $f$ is not continuous everywhere on $[0, T]$, then in general we do not get convergence of the Riemann sum approximations in (1.1) (or the left-most sum in (1.2)) much less convergence of the Riemann step functions in (1.3).

Given a Banach space $(X,\|\cdot\|)$, consider now the space $L^{1}(0, T ; X)$ of all $X$ valued, Bochner integable functions $f$ defined a.e. on the interval $[0, T]$, and having norm

$$
\|f\|_{L^{1}(0, T ; X)}=\int_{0}^{T}\|f(t)\| d t .
$$

Received by the editors October 8, 1984 .

1980 Mathematics Subject Classification (1985 Revision). Primary 28B05.

${ }^{1}$ Supported in part by a grant from the Vanderbilt University Research Council. 
Extend each such $f$ to a Bochner integrable function on all of $\mathbf{R}$ by defining $f \equiv 0$ outside of $[0, T]$. If $f$ belongs to $L^{1}(0, T ; X)$ and is continuous, then the Banach space analogue of (1.3) holds. That is, for any sequence of partitions with mesh size approaching zero and any choice of intermediate points, the functions $w_{n}$ as formed in (1.3) satisfy

$$
\lim _{n \rightarrow \infty}\left\|w_{n}-f\right\|_{L^{1}(0, T ; X)}=0 .
$$

If $f$ is an arbitrary element of $L^{1}(0, T ; X)$, it is well known that $f$ can be approximated in the $L^{1}(0, T ; X)$ norm by step functions of the form $\sum_{k=1}^{m} x_{k} I_{A_{k}}$, where $x_{k} \in X$ and the $A_{k}$ are disjoint intervals (e.g. see Brézis [1, p. 138]). In Lemma A.1 of $[\mathbf{7}]$, Kato refines this result by showing that we can choose $x_{k}=f\left(\xi_{k}\right)$ for some point $\xi_{k}$ in $A_{k}$. Specifically, given $f \in L^{1}(0, T ; X)$, Lemma A.1 states that there exists a sequence of partitions of $[0, T]: 0=t_{0}^{n}<t_{1}^{n}<\cdots<t_{N_{n}}^{n}=T$, with mesh size going to zero as $n \rightarrow \infty$, and there exist points $\xi_{k}^{n} \in\left(t_{k-1}^{n}, t_{k}^{n}\right] \cap \operatorname{Dom}(f)$ such that the sequence of Riemann step functions

$$
w_{n}(t)=\sum_{k=1}^{N_{n}} f\left(\xi_{k}^{n}\right) I_{\left(t_{k-1}^{n}, t_{k}^{n}\right]}(t)
$$

satisfies

$$
\lim _{n \rightarrow \infty}\left\|w_{n}-f\right\|_{L^{1}(0, T ; X)}=0 .
$$

Evans, in Lemma 4.1 of $[\mathbf{3}]$, achieves a more structured approximation by requiring the step functions to take values $f\left(\xi_{k}^{n}\right)$ at the right end points $\xi_{k}^{n}=t_{k}^{n}$. However, since $f$ may not be defined at $T$, Evans has $t_{N_{n}}^{n} \leq T$ and has $t_{N_{n}}^{n}$ approaching $T$ as $n \rightarrow \infty$. We emphasize that Brézis, Kato and Evans are not free to choose their sequence of partitions; instead they show the existence of some suitable sequence of partitions.

In the next result, we show that almost any sequence of partitions can be used, so that Bochner integrability is almost as good as continuity for the purpose of approximation by Riemann step functions. More precisely:

THEOREM 1.1. Let $P^{n}=\left\{t_{k}^{n}\right\}_{k=0}^{N_{n}}(n=1,2, \ldots)$ be any sequence of partitions of $[0, T]$ satisfying

(M.1) $\left\|P^{n}\right\| \leq B \mu\left(P^{n}\right)$ for some constant $B$ and all $n$, where

(M.2) $\left\|P^{n}\right\| \rightarrow 0$ as $n \rightarrow \infty$.

$$
\mu\left(P^{n}\right)=\min _{1 \leq k \leq N_{n}}\left(t_{k}^{n}-t_{k-1}^{n}\right),
$$

Then given $f \in L^{1}(0, T ; X)$, there exists a sequence of sets $S_{k}^{n} \subseteq\left(t_{k-1}^{n}, t_{k}^{n}\right]$ having positive measure such that $f$ is defined at all points $t_{k}^{n}-\delta_{n}, k=1, \ldots, N_{n}$, and such that the Riemann step functions for $f$

$$
w_{n}(t)=\sum_{k=1}^{N_{n}} f\left(\xi_{k}^{n}\right) I_{\left(t_{k-1}^{n}, t_{k}^{n}\right]}(t), \quad \xi_{k}^{n} \in S_{k}^{n},
$$

satisfy

$$
\lim _{n \rightarrow \infty}\left\|f-w_{n}\right\|_{L^{1}(0, T ; X)}=0 .
$$

We mention that condition (M.1) above is a mild one. For example, it is satisfied with $B=1$ along any sequence of equally spaced partitions. The proof of Theorem 1.1 rests on the following elementary lemma. 
Lemma 1.1. Let $f$ belong to $L^{1}(0, T ; X)$. Then

(i) $\lim _{c \rightarrow 0} \int_{0}^{T}\|f(r)-f(r-c)\| d r=0$ and

(ii) $\lim _{\varepsilon \rightarrow 0} \frac{1}{\varepsilon} \int_{0}^{T} \int_{r-\varepsilon}^{r+\varepsilon}\|f(t)-f(r)\| d t d r=0$.

Proof. (i) See [6, p. 86]. (ii) Set $h_{\varepsilon}(\lambda)$ equal to $\int_{0}^{T}\|f(r-[2 \lambda / T-1] \varepsilon)-f(r)\| d r$. Then, by (i) and the Dominated Convergence Theorem, we have $\lim _{\varepsilon \downarrow 0} \int_{0}^{T} h_{\varepsilon}(\lambda) d \lambda$ $=0$. Now substitute $t=r-[2 \lambda / T-1] \varepsilon$.

PROOF OF THEOREM 1.1. Set $\mu_{n}=\mu\left(P^{n}\right)$ and define

$$
J_{n}=\frac{1}{\mu_{n}} \int_{0}^{\mu_{n}} \sum_{k=1}^{N_{n}} \int_{t_{k-1}^{n}}^{t_{k}^{n}}\left\|f(t)-f\left(t_{k}^{n}-\delta\right)\right\| d t d \delta .
$$

Under the substitutions $r=t_{k}^{n}-\delta$, since

$$
t_{k-1}^{n} \geq t_{k-1}^{n}-\delta=\left(t_{k}^{n}-\delta\right)-\left(t_{k}^{n}-t_{k-1}^{n}\right) \geq r-B \mu_{n} \quad \text { and } \quad t_{k}^{n}-\mu_{n} \geq t_{k-1}^{n},
$$

we have

$J_{n} \leq \sum_{k=1}^{N_{n}} \frac{1}{\mu_{n}} \int_{t_{k-1}^{n}}^{t_{k}^{n}} \int_{r-B \mu_{n}}^{r+\mu_{n}}\|f(t)-f(r)\| d t d r=\frac{1}{\mu_{n}} \int_{0}^{T} \int_{r-B \mu_{n}}^{r+\mu_{n}}\|f(t)-f(r)\| d t d r$.

Therefore, there exist sets of positive measure $S_{n} \subseteq\left(0, \mu_{n}\right)$, for which $\delta_{n} \in S_{n}$ implies

$$
\sum_{k=1}^{N_{n}} \int_{t_{k-1}^{n}}^{t_{k}^{n}}\left\|f(t)-f\left(t_{k}^{n}-\delta_{n}\right)\right\| d t \leq \frac{1}{\mu_{n}} \int_{0}^{T} \int_{r-B \mu_{n}}\|f(t)-f(r)\| d t d r .
$$

For each $k$ let $S_{k}^{n}=t_{k}^{n}-S_{n}$. Then with $w_{n}(t)$ as in (1.4) we obtain from (1.6) and Lemma 1.1,

$$
\lim _{n \rightarrow \infty} \int_{0}^{T}\left\|f(t)=w_{n}(t)\right\| d t=0
$$

COROLLARY 1.1. If $f \in L^{1}(0, T ; X)$, then for every sequence of partitions $P^{n}=\left\{t_{k}^{n}\right\}_{k=0}^{N_{n}}$ satisfying conditions (M.1) and (M.2) above, there exists a sequence of points $\xi_{k}^{n} \in\left(t_{k-1}^{n}, t_{k}^{n}\right]$ of the form $\xi_{k}^{n}=t_{k}^{n}-\delta_{n}, k=1, \ldots, N_{n}$, such that

$$
\int_{0}^{T} f(t) d t=\lim _{n \rightarrow \infty} \sum_{k=1}^{N_{n}} f\left(\xi_{k}^{n}\right)\left(t_{k}^{n}-t_{k-1}^{n}\right) .
$$

While from the proof of Theorem 1.1 we see that the set of allowable $\xi_{k}^{n}$ has positive measure, Corollary 1.1 does not hold true in general for almost every $\xi_{k}^{n} \in\left(t_{k-1}^{n}, t_{k}^{n}\right)$. Consider

$$
f(t)=\sum_{n=1}^{\infty} n^{2} I_{\Delta_{n}}(t), \quad \text { where } \Delta_{n}=\left(\frac{T}{n}, \frac{T}{n}+\frac{T}{n^{4}}\right] .
$$

Then along the sequence of partitions $t_{k}^{n}=k T / n$, for all $\xi_{1}^{n} \in \Delta_{n}$ we have

$$
\sum_{k=1}^{n} f\left(\xi_{k}^{n}\right) \frac{T}{n} \geq f\left(\xi_{1}^{n}\right) \frac{T}{n}=n T \rightarrow \infty .
$$

However, the following does hold. 
THEOREM 1.2. Let $P^{n}=\left\{t_{k}^{n}\right\}_{k=0}^{N_{n}}(n=1,2, \ldots)$ be any sequence of partitions of $[0, T]$ satisfying conditions (M.1) and (M.2) and let $\xi_{k}^{n} \in\left(t_{k-1}^{n}, t_{k}^{n}\right]$ be any sequence of intermediate points.

Given $f \in L^{1}(0, T ; X)$, define for a.e. $\theta \in(0, T)$ the Riemann step function

$$
w_{n}^{\theta}(t)=\sum_{k=1}^{N_{n}} f\left(\xi_{k}^{n}-\theta\right) I_{\left(t_{k-1}^{n}-\theta, t_{k}^{n}-\theta\right]}(t) .
$$

Then there exists a subsequence $n(i)$ of the positive integers such that

$$
\lim _{i \rightarrow \infty}\left\|f-w_{n(i)}^{\theta}\right\|_{L^{1}(0, T-\theta ; X)}=0 \text { for a.e. } \theta \in(0, T) .
$$

Proof. Let $\tilde{f}$ denote the periodic extension of $f$. Thus $\tilde{f}(t \pm T)=f(t)$ for almost every $t \in[0, T]$. Define

$$
\mathcal{K}_{n}=\int_{0}^{T} \sum_{k=1}^{N_{n}} \int_{t_{k-1}^{n}-\theta}^{t_{k}^{n}-\theta}\left\|\tilde{f}(t)-\tilde{f}\left(\xi_{k}^{n}-\theta\right)\right\| d t d \theta .
$$

The substitution $r=\xi_{k}^{n}-\theta$ and condition (M.1) give

$$
\begin{aligned}
\mathcal{K}_{n} & =\sum_{k=1}^{N_{n}} \int_{\xi_{k}^{n}-T}^{\xi_{k}^{n}} \int_{r-\left(\xi_{k}^{n}-t_{k-1}^{n}\right)}^{r+\left(t_{k}^{n}-\xi_{k}^{n}\right)}\|\tilde{f}(t)-\tilde{f}(r)\| d t d r \\
& \leq \sum_{k=1}^{N_{n}} \int_{\xi_{k}^{n}-T}^{\xi_{k}^{n}} \int_{r-B \mu_{n}}^{r+B \mu_{n}}\|\tilde{f}(t)-\tilde{f}(r)\| d t d r,
\end{aligned}
$$

where $\mu_{n}=\mu\left(P^{n}\right)$. Next, we make use of the periodicity of $\tilde{f}$. In fact, after working through a number of routine steps the reader may verify

$$
\begin{aligned}
\mathcal{K}_{n} \leq & \sum_{k=1}^{N_{n}} \int_{0}^{T} \int_{r-B \mu_{n}}^{r+B \mu_{n}}\|f(t)-f(r)\| d t d r \\
& +\sum_{k=1}^{N_{n}} \mu_{n}\left[\int_{0}^{B \mu_{n}}\|f(t)\| d t+\int_{T-B \mu_{n}}^{T}\|f(t)\| d t\right] \\
= & N_{n} \int_{0}^{T} \int_{r-B \mu_{n}}^{r+B \mu_{n}}\|f(t)-f(r)\| d t d r \\
& +B N_{n} \mu_{n}\left[\int_{0}^{B \mu_{n}}\|f(t)\| d t+\int_{T-B \mu_{n}}^{T}\|f(t)\| d t\right] \\
\leq & \frac{1}{\mu_{n}} \int_{0}^{T} \int_{r-B \mu_{n}}^{r+B \mu_{n}}\|f(t)-f(r)\| d t d r \\
& +B\left[\int_{0}^{B \mu_{n}}\|f(t)\| d t+\int_{T-B \mu_{n}}^{T}\|f(t)\| d t\right],
\end{aligned}
$$

which, by Lemma 1.1, approaches zero as $n \rightarrow \infty$. Using the fact that $L^{1}$ convergence implies a.e. convergence along some subsequence, we conclude that there exists a subsequence $n(i)$ of the positive integers such that

$$
\lim _{i \rightarrow \infty} \sum_{k=1}^{N_{n(i)}} \int_{t_{k-1}^{n(i)}-\theta}^{t_{k}^{n(i)}-\theta}\left\|\tilde{f}(t)-\tilde{f}\left(\xi_{k}^{n(i)}-\theta\right)\right\| d t=0 \quad \text { for a.e. } \theta \in(0, T) .
$$


Given $c>0$ and $\theta \in(0, T)$ such that (1.8) holds, let $i_{0}$ be such that for all $i \geq i_{0}$,

$$
\sum_{k=1}^{N_{n(i)}} \int_{t_{k-1}^{n(i)}-\theta}^{t_{k}^{n(i)}-\theta}\left\|\tilde{f}(t)-\tilde{f}\left(\xi_{k}^{n(i)}-\theta\right)\right\| d t \leq c
$$

and for all $r$,

$$
\int_{r}^{r+\left\|P^{n(i)}\right\|}\|f(t)\| d t \leq c .
$$

For each $i \geq i_{0}$, let $\underline{k}=\underline{k}(i)$ be such that $t_{\underline{k}-1}^{n(i)}-\theta<0 \leq t_{\underline{k}}^{n(i)}-\theta$. Let

$$
W_{i}=\int_{t_{\underline{k}-1}^{n(i)}-\theta}^{t_{\underline{k}}^{n(i)}-\theta}\left\|f(t)-f\left(\xi_{\underline{k}}^{n(i)}-\theta\right)\right\| d t
$$

and

$$
\tilde{W}_{i}=\int_{t_{\underline{k}-1}^{n(i)}-\theta}^{t_{\underline{k}}^{n(i)}-\theta}\left\|\tilde{f}(t)-\tilde{f}\left(\xi_{\underline{k}}^{n(i)}-\theta\right)\right\| d t .
$$

Suppose $\xi_{\underline{k}}^{n(i)}-\theta<0$. Then by $(1.10), W_{i} \leq c$. On the other hand, if $\xi_{\underline{k}}^{n(i)}-\theta \geq 0$, then by (1.9) and (1.10)

$$
\begin{aligned}
W_{i} & \leq\left|W_{i}-\tilde{W}_{i}\right|+\tilde{W}_{i} \leq \int_{t_{\underline{k}-1}^{n(i)}-\theta}^{t_{\underline{k}}^{n(i)}-\theta}\|\tilde{f}(t)-f(t)\| d t+\tilde{W}_{i} \\
& \leq \int_{t_{\underline{k}-1}^{n(i)}-\theta}^{t_{\boldsymbol{k}}^{n(i)}-\theta}(\|\tilde{f}(t)\|+\|f(t)\|) d t+\tilde{W}_{i} \leq 3 c .
\end{aligned}
$$

In either case, using (1.8) we obtain

$$
\begin{aligned}
\int_{0}^{T-\theta}\left\|f(t)-w_{n(i)}^{\theta}(t)\right\| d t & \leq \sum_{k=1}^{N_{n(i)}} \int_{t_{k-1}^{n(i)}-\theta}^{t_{k}^{n(i)}-\theta}\left\|f(t)-f\left(\xi_{k}^{n(i)}-\theta\right)\right\| d t \\
& =\sum_{k=\underline{k}}^{N_{n(i)}} \int_{t_{k-1}^{n(i)}-\theta}^{t_{k}^{n(i)}-\theta}\left\|\tilde{f}(t)-\tilde{f}\left(\xi_{k}^{n(i)}-\theta\right)\right\| d t+W_{i} \leq 4 c .
\end{aligned}
$$

COROLLARY 1.2. If $f \in L^{1}(0, T ; X)$, then for every sequence of partitions $P^{n}=\left\{t_{k}^{n}\right\}_{k=0}^{N_{n}}$ satisfying (M.1) and (M.2) and every sequence of intermediate points $\xi_{k}^{n} \in\left(t_{k-1}^{n}, t_{k}^{n}\right]$, there exists a subsequence $n(i)$ of the positive integers such that

$$
\int_{0}^{T-\theta} f(t) d t=\lim _{i \rightarrow \infty} \sum_{k=1}^{N_{n(i)}} f\left(\xi_{k}^{n(i)}-\theta\right)\left(t_{k}^{n(i)}-t_{k-1}^{n(i)}\right) \quad \text { for a.e. } \theta \in(0, T) .
$$

REMARKS. 1. The proof of the preceding theorem shows that if the function $f$ is replaced by $\tilde{f}$ in the formulation of $w_{n}^{\theta}(t)$, then the conclusion of Theorem 1.2 can be strengthened to

$$
\lim _{i \rightarrow \infty}\left\|f-w_{n(i)}^{\theta}\right\|_{L^{1}(0, T ; X)}=0 \text { for a.e. } \theta \in(0, T),
$$


and (1.11) can be changed to

$$
\int_{0}^{T} f(t) d t=\lim _{i \rightarrow \infty} \sum_{k=1}^{N_{n(i)}} \tilde{f}\left(\xi_{k}^{n(i)}-\theta\right)\left(t_{k}^{n(i)}-t_{k-1}^{n(i)}\right) \quad \text { for a.e. } \theta \in(0, T) .
$$

2. Theorems 1.1 and 1.2 and Corollaries 1.1 and 1.2 can be strengthened so that the convergence is uniform on $[0, T]$. For example, the conclusion to Corollary 1.1 can be strengthened to

$$
\int_{\sigma}^{\tau} f(t) d t=\lim _{n \rightarrow \infty} \sum_{k=q(\sigma, n)}^{q(\tau, n)} f\left(\xi_{k}^{n}\right)\left(t_{k}^{n}-t_{k-1}^{n}\right) \quad \text { uniformly for all } \sigma, \tau \in[0, T],
$$

where $q(\xi, n)$ is that index such that $\xi \in\left(t_{q(\xi, n)-1}^{n}, t_{q(\xi, n)}^{n}\right]$. The proofs follow readily using the above techniques together with the absolute continuity property of the Bochner integral.

3. It may be shown that all the above results hold equally true for arbitrary functions in $L^{p}(0, T ; X)$ for any $1 \leq p<\infty$.

2. Convergence of the product integral. This section is concerned with the initial value problem

$$
\frac{d u}{d t}(t)+A(t) u(t) \ni 0, \quad 0 \leq t \leq T, \quad u(0)=x \in X,
$$

where $u$ takes values in an arbitrary Banach space $X$ and for each $t, A(t)$ is a nonlinear, possibly multivalued operator. Consider the following theorem.

THEOREM 2.1 (CRANDALl AND PAZY [2]). Assume that for some real number $\omega$, the family of operators $A(t), 0 \leq t \leq T$, satisfies

(Q.1) $\bar{D}=\overline{\operatorname{Dom}(A(t))}$ is independent of $t$,

(Q.2) $\operatorname{Ran}(I+\lambda A(t)) \supseteq \bar{D}$ for $0 \leq t \leq T$ and $0<\lambda<\lambda_{0}$, where $\omega \lambda_{0}<1$, and

(Q.3) Each resolvent operator $J_{\lambda}(t)=[I+\lambda A(t)]^{-1}$ is a Lipschitz mapping on $\bar{D}$ with $\left\langle J_{\lambda}(t)\right\rangle_{\text {Lip }} \leq(1-\omega \lambda)^{-1}$.

In addition, assume that $A(t)$ is restricted by one of the following time-dependence conditions:

(C.1) There exist a continuous function $h:[0, T] \rightarrow X$ and an increasing continuous function $L$ such that

$$
\left\|J_{\lambda}(t) x-J_{\lambda}(\tau) x\right\| \leq \lambda\|h(t)-h(\tau)\| L(\|x\|)
$$

for $0<\lambda<\lambda_{0}, 0<t, \tau \leq T$ and $x \in \bar{D}$;

(C.2) There exist a continuous function $h:[0, T] \rightarrow X$ of bounded variation and an increasing continuous function $L$ such that

$$
\left\|J_{\lambda}(t) x-J_{\lambda}(\tau) x\right\| \leq \lambda\|h(t)-h(\tau)\| L(\|x\|)\left\{1+\left\|A(\tau) J_{\lambda}(\tau) x\right\|\right\}
$$

for $0<\lambda<\lambda_{0}, 0<t, \tau \leq T$ and $x \in \bar{D}$.

If, given $x \in \bar{D}$, the initial value problem (IVP) has a strong solution $u(t)$ on $[0, T]$, then for each $t \in(0, T]$, the product integral

$$
\lim _{n \rightarrow \infty} \prod_{k=1}^{n} J_{t / n}(k t / n) x
$$

exists and equals $u(t)$ uniformly on $[0, T]$. 
By a strong solution of (IVP) on $[0, T]$, Crandall and Pazy mean a function $u:[0, T] \rightarrow X$ for which

(S.1) $u$ is continuous on $[0, T]$ and $u(0)=x$,

(S.2) $u$ is absolutely continuous on compact subsets of $(0, T)$,

(S.3) $u$ is differentiable a.e. on $(0, T)$ and satisfies the differential equation of (IVP) a.e.

However, for our purposes, throughout the remainder of this section we shall replace (S.2) with the stronger hypothesis

$\left(\mathrm{S} .2^{\prime}\right) u$ is absolutely continuous on $[0, T]$.

Note that the convergence in (PI) is along the sequence of equally spaced partitions of $[0, t]: 0=t_{0}^{n}<t_{1}^{n}<\cdots<t_{n}^{n}=t$, where $t_{k}^{n}=k t / n$. However, since the modulus function $h(t)$ in (C.1) and (C.2) is continuous, it can be shown that for every sequence of partitions $P^{n}: 0=t_{0}^{n}<t_{1}^{n}<\cdots<t_{N_{n}}^{n}=T$ with $\left\|P^{n}\right\|$ approaching zero and every sequence of intermediate points $\xi_{k}^{n} \in\left[t_{k-1}^{n}, t_{k}^{n}\right]$, the product integral

$$
\lim _{n \rightarrow \infty} \prod_{k=1}^{q(t, n)} J_{t_{k}^{n}-t_{k-1}^{n}}\left(\xi_{k}^{n}\right) x
$$

exists and equals $u(t)$ uniformly on $[0, T]$.

The next result, based on Evans [3, pp. 32, 33], eliminates the need for time dependence restrictions like (C.1) or (C.2).

TheOREM 2.2 (EvANS). Assume (Q.1)-(Q.3) for almost every $t, \tau \in[0, \bar{T}]$. Let $x \in \bar{D}$ be given. Suppose (IVP) has a strong solution $u(t)$ on $[0, \bar{T}]$. Then for every $0<T<\bar{T}$, there are partitions $P^{n}: 0=t_{0}^{n}<t_{1}^{n}<\cdots<t_{N_{n}}^{n}$ such that $T \leq t_{N_{n}}^{n} \leq \bar{T}, \lim _{n \rightarrow \infty}\left\|P^{n}\right\|=0$ and when $\xi_{k}^{n} \equiv t_{k}^{n}$, the product integral $\left(\mathrm{PI}^{\prime}\right)$ exists and equals $u(t)$ uniformly on $[0, T]$.

In contrast to Theorem 2.1, in general in Theorem 2.2 we cannot expect to have convergence of the product integral along every sequence of partitions with mesh size approaching zero. For along every such sequence of partitions, the family of resolvents operators which comprise the finite product aproximation to the product integral in $\left(\mathrm{PI}^{\prime}\right)$ can be redefined without changing the hypotheses of the theorem.

In the next theorem, however, we are able to achieve convergence of the product integral along every sequence of partitions satisfying (M.1) and (M.2), much as in $\left(\mathrm{PI}^{\prime}\right)$. The proof follows from Theorem 1.1 using the technique of Theorem 2.2 and is left to the reader.

THEOREM 2.3. Given $x \in \bar{D}$, suppose (IVP) has a strong solution $u(t)$ on $[0, T]$. Further assume that the family of operators $A(t)$ satisfies (Q.1)-(Q.3) almost everywhere on $[0, T]$. Let $P^{n}=\left\{t_{k}^{n}\right\}_{k=0}^{N_{n}}$ be any sequence of partitions of $[0, T]$ satisfying (M.1) and (M.2). Then there exist points $\xi_{k}^{n} \in\left(t_{k-1}^{n}, t_{k}^{n}\right]$ of the form $\xi_{k}^{n}=t_{k}^{n}-\delta_{n}, k=1, \ldots, N_{n}$, such that $\left(\mathrm{PI}^{\prime}\right)$ exists and equals $u(t)$ uniformly on $[0, T]$. 
Likewise, using Theorem 1.2 we may prove

THEOREM 2.4. Under the hypotheses of Theorem 2.3, for every sequence of intermediate points $\xi_{k}^{n} \in\left(t_{k-1}^{n}, t_{k}^{n}\right]$, there exists a subsequence $n(i)$ of the positive integers such that

$$
u(t-\theta)=\lim _{i \rightarrow \infty} \prod_{i=1}^{q(t, n(i))} J_{t_{k}^{n(i)}-t_{k-1}^{n(i)}}\left(\xi_{k}^{n(i)}-\theta\right) x
$$

for a.e. $\theta \in(0, t)$ uniformly on $(0, T]$.

REMARKS. 1. Observe that Theorem 2.3 generalizes Theorem 2.2.

2. Theorem 2.3 will not hold true, in general, for almost every $\xi_{k}^{n} \in\left(t_{k-1}^{n}, t_{k}^{n}\right]$. Consider $A(t)=f(t)$ as defined by (1.7). Then the strong solution to (IVP) will be $u(t)=\exp \left(-\int_{0}^{t} A(s) d s\right) u(0)$. In particular, $u(T)=\exp \left(-T \sum_{n=1}^{\infty} 1 / n^{2}\right) u(0) \neq 0$ for $u(0) \neq 0$. However, along the sequence of equally spaced partitions of $[0, T]$, namely $t_{k}^{n}=k T / n$, we have

$\left|\prod_{k=1}^{n} J_{T / n}\left(\xi_{k}^{n}\right) u(0)\right| \leq\left|\left[1+(T / n) A\left(\xi_{1}^{n}\right)\right]^{-1}\right||u(0)|=\frac{1}{1+T n}|u(0)| \rightarrow 0 \quad$ as $n \rightarrow \infty$, for all $\xi_{1}^{n} \in\left(T / n, T / n+T / n^{4}\right]$.

3. In Theorem 2.1 of [2], it is shown that properties (Q.1)-(Q.3) and either (C.1) or (C.2) imply existence of the product integral (PI). In fact, in their proof of the above-stated Theorem 2.1, Crandall and Pazy make use of this existence. However, as discussed below, (Q.1)-(Q.3) alone are not enough to ensure convergence in (PI). An additional hypothesis, such as existence of strong solution to (IVP), is required. Therefore, Theorems $2.2,2.3$ and 2.4 provide a new sufficient condition for existence of the product integral. This condition is exploited in $[5]$ in connection with proving existence of strong solution to singular evolution equations.

4. As a special case of Theorem 2.3, consider when $A(t)$ satisfies (Q.1)-(Q.3) and nearly satisfies either (C.1) or (C.2). That is, in place of (C.1) or (C.2), $A(t)$ is restricted by either $\left(\mathrm{C} .1^{\prime}\right)$ or $\left(\mathrm{C} .2^{\prime}\right)$ given by

$\left(\mathrm{C} .1^{\prime}\right),\left(\mathrm{C} .2^{\prime}\right):$ the same as (C.1) and (C.2), respectively, on (0,T]. At $t=0, h(t)$ need not be continuous, but we require that the map $(\lambda, s, x):\left[0, \lambda_{0}\right] \times[0, T] \times \bar{D} \mapsto$ $J_{\lambda}(s) x$ be continuous.

The distinction between (C.1), (C.2) and (C.1'), (C.2') may seem insignificant. However, one point made in [4] is that there exist generators $A(t)$ having all their pathologies concentrated at $t=0$, so that the distinction can be crucial with respect to existence of the product integral. For, as shown in [4], properties (Q.1j-(Q.3) and either $\left(\mathrm{C} .1^{\prime}\right)$ or $\left(\mathrm{C} .2^{\prime}\right)$ do not in themselves guarantee convergence of (PI) or even of $\left(\mathrm{PI}^{\prime}\right)$ for any sequence of partitions $P^{n}$ with mesh size approaching zero.

\section{REFERENCES}

1. H. Brézis, Opérateurs maximaux monotones, North-Holland, Amsterdam, 1973.

2. M. G. Crandall and A. Pazy, Nonlinear evolution equations in Banach spaces, Israel J. Math. 11 (1972), 57-94.

3. L. C. Evans, Nonlinear evolution equations in an arbitrary Banach space, Israel J. Math. 26 (1977), 1-42. 
4. M. A. Freedman, Product integrals of continuous resolvents: existence and nonexistence, Israel J. Math. 46 (1983), 145-160.

5. __ Existence of strong solutions to singular nonlinear evolution equations, Pacific J. Math. 120 (1985), 331-344.

6. E. Hille and R. Phillips, Functional analysis and semi-groups, rev. ed., Amer. Math. Soc. Colloq. Publ., vol. 31, Amer. Math. Soc., Providence, R.I., 1957.

7. T. Kato, Linear evolution equations of "hyperbolic" type. II, J. Math. Soc. Japan 25 (1973), 648-666.

Department of Mathematics, Vanderbilt University, NashVille, TenNessee 37235

Current address: Department of Mathematical Sciences, University of Alaska, Fairbanks, Alaska 99775 